\title{
Robust Filtering With Randomly Varying Sensor Delay: The Finite-Horizon Case
}

\author{
Fuwen Yang, Senior Member, IEEE, Zidong Wang, Senior Member, IEEE, Gang Feng, Fellow, IEEE, and
} Xiaohui Liu

\begin{abstract}
In this paper, we consider the robust filtering problem for discrete time-varying systems with delayed sensor measurement subject to norm-bounded parameter uncertainties. The delayed sensor measurement is assumed to be a linear function of a stochastic variable that satisfies the Bernoulli random binary distribution law. An upper bound for the actual covariance of the uncertain stochastic parameter system is derived and used for estimation variance constraints. Such an upper bound is then minimized over the filter parameters for all stochastic sensor delays and admissible deterministic uncertainties. It is shown that the desired filter can be obtained in terms of solutions to two discrete Riccati difference equations of a form suitable for recursive computation in online applications. An illustrative example is presented to show the applicability of the proposed method.
\end{abstract}

Index Terms-Kalman filtering, parameter uncertainty, random sensor delay, robust filtering, time-varying systems.

\section{INTRODUCTION}

$\mathbf{K}$ ALMAN filtering has proven to be very popular in a number of research areas such as signal processing and communication [1]. Guaranteeing the robust performance of Kalman filters (especially in the presence of system parameter uncertainties) has become an important research topic primarily due to the Kalman filters' sensitivity to model structure drift [1]. A large volume of literature has been published on the general topic of robust and/or $H_{\infty}$ filtering problems for systems with various parameter uncertainties; see, for example, [2], [5]-[7], [9]-[11], [14], [15], [17], [20]-[22], [25], [26], [28], [32] and the references therein.

There is an implicit assumption with the Kalman filtering approach that sensor data which may or may not be corrupted by noise always contains information about the current state of the

Manuscript received September 21, 2004; revised July 13, 2007. Current version published March 11, 2009. This work was supported in part by the Engineering and Physical Sciences Research Council (EPSRC) of the U.K. under Grant GR/S27658/01, the National 973 Program of China under Grant 2009CB320600, the Nature Science Foundation of Fujian Province of China under Grants A0510009 and 2007J0018, and the Alexander von Humboldt Foundation of Germany. This paper was recommended by Associate Editor L. Trajkovic.

F. Yang was with the Department of Information Systems and Computing, Brunel University, Uxbridge, Middlesex, UB8 3PH, U.K. He is now with the School of Information Science and Engineering, East China University of Science and Technology, Shanghai 200237, China.

Z. Wang and X. Liu are with the Department of Information Systems and Computing, Brunel University, Uxbridge, Middlesex, UB8 3PH, U.K. (e-mail: Zidong.Wang@brunel.ac.uk).

G. Feng is with the Department of Manufacturing Engineering and Engineering Management, City University of Hong Kong, Kowloon, Hong Kong.

Digital Object Identifier 10.1109/TCSI.2008.918009 system. However, this is not always the case in engineering, biological, and economic systems, where system measurements (or outputs) may be delayed. These delays could cause performance degradation or instability with traditional Kalman filters [12], [13]. Therefore, as can be seen in [4], [8], [16], [19], [23], [29], [31], the filtering problem with delayed measurements has received a great deal of research interest. However, most of the publications assume that time delays in the measurement are always deterministic. Unfortunately, time delays may occur in a random way for a large class of practical applications. For example, in real-time distributed decision-making and multiplexed data communication networks, the measurement device or sensor is often randomly delayed. Alternatively, the measurements are interrupted such that the measurements available to the predictor mechanism are not up to date [19], [23], [30]. Hence, there is a need to develop new filtering methods for signal processing problems in these delayed environments for general network-based systems.

Recently, there have been several papers discussing the filter design issue with randomly varying delayed measurements. In [30], a linear unbiased state estimation problem has been examined for discrete-time systems with random sensor delays over both finite and infinite horizons where the full and reduced-order filters were designed to achieve specific estimation error covariances. The results of [30] were extended in [23] to the case where parameter uncertainties (or modeling error) were taken into account. However, in [23], only the stationary (or infinite-horizon) robust filtering problem has been studied. It is well known that finite-horizon filters could provide a better transient performance for filtering process systems where noise inputs are nonstationary. Our aim in this paper is therefore to further study the finite-horizon counterpart of [23]. That is, we intend to tackle the finite-horizon filtering problem for uncertain discrete time-varying systems subject to both randomly varying sensor delays and parameter uncertainties. Unlike the work of [23], here the nominal system is also allowed to be time-varying, and an optimization approach based on the solutions to two discrete Riccati difference equations is used.

In this paper, we are concerned with the robust filtering problem for discrete time-varying systems with delayed sensor measurements subject to norm-bounded parameter uncertainties. The delayed sensor measurement is assumed to be a linear function of a stochastic variable that satisfies the Bernoulli random binary distribution law. An upper bound for the actual covariance of uncertain stochastic parameter systems is derived and used for the estimation variance constraints. Such an upper bound is then minimized over the filter parameters for all 
stochastic sensor delays and admissible deterministic uncertainties. This unfortunately renders the filter design problem suboptimal. A Riccati difference equation approach is developed to design the expected filter parameters. Such an approach is suitable for recursive computation in online applications. We illustrate the applicability of the proposed method by means of a simulation example.

The remainder of this paper is organized as follows. The robust suboptimal filter design problem is formulated in Section II for uncertain discrete-time systems subject to random sensor delays. The covariance of uncertain stochastic parameter systems is derived and the upper bound is provided in Section III. Sufficient conditions for filter design are developed in Section IV such that the upper bound state estimation error variance is guaranteed while simultaneously minimized. A simulation result is given in Section $\mathrm{V}$ to demonstrate the effectiveness of the proposed method. Some concluding remarks are provided in Section VI.

\section{Notation}

The notation used here is fairly standard. $\mathbb{R}^{n}$ and $\mathbb{R}^{n \times m}$ denote the $n$-dimensional Euclidean space and the set of all $n \times m$ real matrices, respectively. The notation $X \geq Y$ (respectively, $X>Y$ ), where $X$ and $Y$ are symmetric matrices, means that $X-Y$ is positive semi-definite (respectively, positive definite). $A^{i i}$ denotes diagonal block submatrix of matrix $A$ with respect to the $i$ th row and $i$ th column. $x^{i}$ represents the $i$ th element of vector $x$. $\operatorname{Cov}(x)$ means the covariance of $x$. The superscript " $T$ " denotes the transpose. $\mathbb{E}\{x\}$ stands for the expectation of $x$. $\operatorname{Prob}\{\cdot\}$ means the occurrence probability of the event ".". The arguments of a function will be omitted in the analysis sometimes where no confusion should arise.

\section{PROBLEM Formulation AND PRELIMINARIES}

Consider a class of uncertain linear discrete time-varying systems

$$
\vec{x}_{k+1}=\left(\vec{A}_{k}+\Delta \vec{A}_{k}\right) \vec{x}_{k}+\vec{B}_{k} w_{k}
$$

where $\vec{x}_{k} \in \mathbb{R}^{n}$ is a state vector and $w_{k} \in \mathbb{R}^{m}$ is a zero-mean Gaussian white noise sequence with covariance $Q_{k}>0$. The delayed sensor measurement is described by

$$
\begin{aligned}
& \vec{y}_{k}=\vec{C}_{k} \vec{x}_{k}+\vec{v}_{k} \\
& y_{k}=\left(1-\gamma_{k}\right) \vec{y}_{k}+\gamma_{k} \vec{y}_{k-1}
\end{aligned}
$$

where $\vec{y}_{k} \in \mathbb{R}^{p}$ is an actual output vector, $y_{k} \in \mathbb{R}^{p}$ is a measured output vector, and $\vec{v}_{k} \in \mathbb{R}^{p}$ a zero-mean Gaussian white noise sequence with covariance $\vec{R}_{k}>0$ uncorrelated with $w_{k}$. The initial state $\vec{x}_{0}$ has the mean $\bar{x}_{0}$ and covariance $P_{0}$ and is uncorrelated with either $w(k)$ or $\vec{v}(k) . \vec{A}_{k}, \vec{B}_{k}$, and $\overrightarrow{C_{k}}$ are known real time-varying matrices with appropriate dimensions. $\Delta \overrightarrow{A_{k}}$ is a real-valued uncertain matrix satisfying

$$
\Delta \vec{A}_{k}=\vec{H}_{k} F_{k} \vec{E}_{k}, \quad F_{k} F_{k}^{T} \leq I
$$

Here, $\vec{H}_{k}$ and $\vec{E}_{k}$ are known time-varying matrices of appropriate dimensions and $F_{k}$ represents time-varying uncertainties.
The parameter uncertainty in $\Delta \vec{A}_{k}$ is said to be admissible if (4) holds.

The stochastic variable $\gamma_{k} \in \mathbb{R}$ is a Bernoulli distributed white sequence taking values on 0 or 1 with

$$
\operatorname{Prob}\left\{\gamma_{k}=1\right\}=\mathbb{E}\left\{\gamma_{k}\right\}:=\beta_{k}
$$

where $\beta_{k} \in \mathbb{R}$ is a known time-varying positive scalar and $\gamma_{k} \in$ $\mathbb{R}$ is assumed to be independent of $w_{k}, \vec{v}_{k}$, and $\vec{x}_{0}$. Therefore, we have

$$
\begin{aligned}
\operatorname{Prob}\left\{\gamma_{k}=0\right\} & =1-\beta_{k} \\
\sigma_{\gamma}^{2} & :=\mathbb{E}\left\{\left(\gamma_{k}-\beta_{k}\right)^{2}\right\} \\
& =\left(1-\beta_{k}\right) \beta_{k} .
\end{aligned}
$$

Remark 1: The system measurement mode (3) was introduced in [19] and has been employed in [23] and [30]. In measurement (3), the output $y_{k}$ produced at time $k$ is sent to the filter via a communication channel and arrives at time $k+t_{d}$. If the sampling period is long compared with $t_{d}$, there is no need to consider the influence of the delay (i.e., $y_{k}=\vec{y}_{k}$ ). If $t_{d}$ is longer than one sampling period and shorter than two sampling periods, the measurement is then $y_{k}=\vec{y}_{k-1}$. It can be seen that, at the $k$ th sampling time, the actual system output takes the value $\vec{y}_{k-1}$ with probability $\beta_{k}$ and the value $\vec{y}_{k}$ with probability $1-\beta_{k}$. Obviously, long time delays would occur if the binary stochastic variable $\gamma_{k}$ takes the value 1 consecutively at different sample times.

By defining

$$
\begin{aligned}
x_{k} & :=\left[\begin{array}{c}
\vec{x}_{k} \\
\vec{x}_{k-1}
\end{array}\right] \\
A_{k} & :=\left[\begin{array}{cc}
\vec{A}_{k} & 0 \\
I_{n} & 0
\end{array}\right] \\
H_{k} & :=\left[\begin{array}{c}
\vec{H}_{k} \\
0
\end{array}\right] \\
E_{k} & :=\left[\begin{array}{cc}
\vec{E}_{k} & 0
\end{array}\right] \\
\Delta A_{k} & :=H_{k} F_{k} E_{k} \\
C_{k}\left(\gamma_{k}\right) & =\left[\begin{array}{ll}
\left(1-\gamma_{k}\right) \vec{C}_{k} & \gamma_{k} \vec{C}_{k-1}
\end{array}\right] \\
B_{k} & =\left[\begin{array}{c}
\vec{B}_{k} \\
0
\end{array}\right] \\
D_{k}\left(\gamma_{k}\right) & =\left[\begin{array}{ll}
\left(1-\gamma_{k}\right) I_{p} & \gamma_{k} I_{p}
\end{array}\right] \\
v_{k} & =\left[\begin{array}{c}
\vec{v}_{k} \\
\vec{v}_{k-1}
\end{array}\right]
\end{aligned}
$$

we combine the uncertain system (1) and the delayed sensor measurement (2)-(3) as follows:

$$
\begin{aligned}
x_{k+1} & =\left(A_{k}+\Delta A_{k}\right) x_{k}+B_{k} w_{k} \\
y_{k} & =C_{k}\left(\gamma_{k}\right) x_{k}+D_{k}\left(\gamma_{k}\right) v_{k}
\end{aligned}
$$

where $v_{k}$ is a zero-mean Gaussian white noise sequence with covariance

$$
R_{k}:=\left[\begin{array}{cc}
\vec{R}_{k} & 0 \\
0 & \vec{R}_{k-1}
\end{array}\right]
$$


and is independent of $w_{k}, \gamma_{k}$, and $\vec{x}_{0}$. Since $C_{k}\left(\gamma_{k}\right)$ and $D_{k}\left(\gamma_{k}\right)$ involve the stochastic variable $\gamma_{k},(12)-(13)$ is actually a stochastic parameter system.

Denoting

$$
\begin{aligned}
& \bar{C}_{k}=\mathbb{E}\left[C_{k}\left(\gamma_{k}\right)\right]=\left[\begin{array}{ll}
\left(1-\beta_{k}\right) \vec{C}_{k} & \beta_{k} \vec{C}_{k-1}
\end{array}\right] \\
& \bar{D}_{k}=\mathbb{E}\left[D_{k}\left(\gamma_{k}\right)\right]=\left[\left(1-\beta_{k}\right) I_{p} \quad \beta_{k} I_{p}\right]
\end{aligned}
$$

we can rewrite (13) as

$$
y_{k}=\bar{C}_{k} x_{k}+\bar{D}_{k} v_{k}+\tilde{C}_{k}\left(\gamma_{k}\right) x_{k}+\tilde{D}_{k}\left(\gamma_{k}\right) v_{k}
$$

where

$$
\begin{aligned}
& \tilde{C}_{k}\left(\gamma_{k}\right):=C_{k}\left(\gamma_{k}\right)-\bar{C}_{k} \\
& =\left[\begin{array}{ll}
\left(\beta_{k}-\gamma_{k}\right) \vec{C}_{k} & \left(\gamma_{k}-\beta_{k}\right) \vec{C}_{k-1}
\end{array}\right] \\
& =\left(\gamma_{k}-\beta_{k}\right)\left[\begin{array}{ll}
-\vec{C}_{k} & \vec{C}_{k-1}
\end{array}\right] \\
& =\left(\gamma_{k}-\beta_{k}\right) C_{e k} \\
& \tilde{D}_{k}\left(\gamma_{k}\right):=D_{k}\left(\gamma_{k}\right)-\bar{D}_{k} \\
& =\left[\begin{array}{ll}
\left(\beta_{k}-\gamma_{k}\right) I_{p} & \left(\gamma_{k}-\beta_{k}\right) I_{p}
\end{array}\right] \\
& =\left(\gamma_{k}-\beta_{k}\right)\left[\begin{array}{ll}
-I_{p} & I_{p}
\end{array}\right] \\
& =\left(\gamma_{k}-\beta_{k}\right) D_{e k} \\
& C_{e k}:=\left[\begin{array}{ll}
-\vec{C}_{k} & \vec{C}_{k-1}
\end{array}\right] \\
& D_{e k}:=\left[\begin{array}{ll}
-I_{p} & I_{p}
\end{array}\right] \text {. }
\end{aligned}
$$

It can be shown that $\tilde{C}_{k}\left(\gamma_{k}\right) \in \mathbb{R}^{p \times 2 n}$ and $\tilde{D}_{k}\left(\gamma_{k}\right) \in \mathbb{R}^{p \times 2 p}$ are zero-mean stochastic matrix sequences. In this paper, a fullorder filter has structure

$$
\hat{x}_{k+1}=\hat{A}_{k} \hat{x}_{k}+\hat{K}_{k}\left(y_{k}-\bar{C}_{k} \hat{x}_{k}\right)
$$

where $\hat{x}_{k} \in \mathbb{R}^{2 n}$ is the state estimate of the stochastic parameter system (12)-(17) and $\hat{A}_{k}$ and $\hat{K}_{k}$ are the filter parameters to be determined.

Remark 2: The system under consideration is both stochastic and uncertain, whereas the designed filter depends on neither stochastic parameters nor parameter uncertainties. In order to facilitate the implementation, the filter considered in this paper is assumed to be linear without delays. It would be interesting to explore in future research the possibility of designing a nonlinear filter that first detects whether delays occur and then proceeds according to the detection. Also, we are currently investigating how to deal with more general systems consisting of multiple sensor delays and possible missing measurements.

The objective of this paper is twofold. First, we intend to design a finite-horizon filter (22) such that there exists a sequence of positive-definite matrices $\Theta_{k}(0<k \leq N)$ satisfying

$$
\mathbb{E}\left[\left(x_{k}-\hat{x}_{k}\right)\left(x_{k}-\hat{x}_{k}\right)^{T}\right] \leq \Theta_{k}, \quad \forall k
$$

that is, a finite upper bound for the estimation error variance is guaranteed. Second, we shall minimize the bound $\Theta_{k}$ in the sense of the matrix norm and then obtain an optimized filter. This problem will be referred to as a finite-horizon robust filtering problem.

\section{COVARIANCE AND UPPER BOUNDS}

It is noted in the last section that system parameters of (17) contain stochastic terms due to delayed sensor measurement. Therefore, we need to derive the estimation error covariance and obtain a corresponding upper bound. For this purpose, we define a new state vector

$$
\tilde{x}_{k}=\left[\begin{array}{l}
x_{k} \\
\hat{x}_{k}
\end{array}\right]
$$

and then an augmented state-space model combining system (12) and filter (22) can be expressed as

$$
\tilde{x}_{k+1}=\left(\tilde{A}_{k}+\tilde{H}_{k} F_{k} \tilde{E}_{k}\right) \tilde{x}_{k}+\tilde{A}_{e k} \tilde{x}_{k}+\tilde{B}_{1 k} w_{k}+\tilde{B}_{2 k} v_{k}+\tilde{B}_{e k} v_{k}
$$

where

$$
\begin{aligned}
\tilde{A}_{k} & =\left[\begin{array}{cc}
A_{k} & 0 \\
\hat{K}_{k} \bar{C}_{k} & \hat{A}_{k}-\hat{K}_{k} \bar{C}_{k}
\end{array}\right] \\
\tilde{H}_{k} & =\left[\begin{array}{c}
H_{k} \\
0
\end{array}\right] \\
\tilde{E}_{k} & =\left[\begin{array}{cc}
E_{k} & 0
\end{array}\right] \\
\tilde{A}_{e k} & =\left[\begin{array}{cc}
0 & 0 \\
\hat{K}_{k} \tilde{C}_{k}\left(\gamma_{k}\right) & 0
\end{array}\right] \\
\tilde{B}_{1 k} & =\left[\begin{array}{c}
B_{k} \\
0
\end{array}\right] \\
\tilde{B}_{2 k} & =\left[\begin{array}{c}
0 \\
\hat{K}_{k} \bar{D}_{k}
\end{array}\right] \\
\tilde{B}_{e k} & =\left[\begin{array}{c}
0 \\
\hat{K}_{k} \tilde{D}_{k}\left(\gamma_{k}\right)
\end{array}\right] .
\end{aligned}
$$

Note that $\tilde{A}_{k}, \tilde{H}_{k}, \tilde{E}_{k}, \tilde{B}_{1 k}$, and $\tilde{B}_{2 k}$ are deterministic parameters and $\tilde{A}_{e k}$ and $\tilde{B}_{e k}$ are stochastic parameters having zero mean values. Hence, the augmented system (25) is a stochastic parameter system. The state covariance matrix of the augmented system (25) can be defined as

$$
\tilde{\Sigma}_{k}:=\mathbb{E}\left[\tilde{x}_{k} \tilde{x}_{k}^{T}\right]=\mathbb{E}\left\{\left[\begin{array}{l}
x_{k} \\
\hat{x}_{k}
\end{array}\right]\left[\begin{array}{l}
x_{k} \\
\hat{x}_{k}
\end{array}\right]^{T}\right\} .
$$

Since $\tilde{A}_{e k}$ and $\tilde{B}_{e k}$ are zero mean stochastic matrix sequences in (25), we have the following Lyapunov equation that governs the evolution of the covariance matrix $\tilde{\Sigma}_{k}$ from (25) as:

$$
\begin{array}{r}
\tilde{\Sigma}_{k+1}=\left(\tilde{A}_{k}+\tilde{H}_{k} F_{k} \tilde{E}_{k}\right) \tilde{\Sigma}_{k}\left(\tilde{A}_{k}+\tilde{H}_{k} F_{k} \tilde{E}_{k}\right)^{T}+\Psi_{k} \\
+\tilde{B}_{1 k} Q_{k} \tilde{B}_{1 k}^{T}+\tilde{B}_{2 k} R_{k} \tilde{B}_{2 k}^{T}+\Phi_{k}
\end{array}
$$

where

$$
\begin{aligned}
\Psi_{k} & :=\mathbb{E}\left[\tilde{A}_{e k} \tilde{\Sigma}_{k} \tilde{A}_{e k}^{T}\right] \\
& =\mathbb{E}\left\{\left[\begin{array}{cc}
0 & 0 \\
\left(\gamma_{k}-\beta_{k}\right) \hat{K}_{k} C_{e k} & 0
\end{array}\right] \tilde{\Sigma}_{k}\left[\begin{array}{cc}
0 & 0 \\
\left(\gamma_{k}-\beta_{k}\right) \hat{K}_{k} C_{e k} & 0
\end{array}\right]^{T}\right\} \\
& =\mathbb{E}\left[\left(\gamma_{k}-\beta_{k}\right)^{2}\right]\left[\begin{array}{cc}
0 & 0 \\
\hat{K}_{k} C_{e k} & 0
\end{array}\right] \tilde{\Sigma}_{k}\left[\begin{array}{cc}
0 & 0 \\
\hat{K}_{k} C_{e k} & 0
\end{array}\right]^{T}
\end{aligned}
$$




$$
\begin{aligned}
& =\left(1-\beta_{k}\right) \beta_{k}\left[\begin{array}{cc}
0 & 0 \\
\hat{K}_{k} C_{e k} & 0
\end{array}\right] \tilde{\Sigma}_{k}\left[\begin{array}{cc}
0 & 0 \\
\hat{K}_{k} C_{e k} & 0
\end{array}\right]^{T} \\
& =\delta_{k}\left[\begin{array}{cr}
0 & 0 \\
\hat{K}_{k} C_{e k} & 0
\end{array}\right] \tilde{\Sigma}_{k}\left[\begin{array}{cc}
0 & 0 \\
\Phi_{k} C_{e k} & 0
\end{array}\right]^{T} \\
& =\mathbb{E}\left[\tilde{B}_{e k} R_{k} \tilde{B}_{e k}^{T}\right] \\
& =\mathbb{E}\left\{\left[\begin{array}{c}
0 \\
\left(\gamma_{k}-\beta_{k}\right) \hat{K}_{k} D_{e k}
\end{array}\right] R_{k}\left[\begin{array}{c}
0 \\
\left(\gamma_{k}-\beta_{k}\right) \hat{K}_{k} D_{e k}
\end{array}\right]^{T}\right\} \\
& =\mathbb{E}\left[\left(\gamma_{k}-\beta_{k}\right)^{2}\right]\left[\begin{array}{c}
0 \\
\hat{K}_{k} D_{e k}
\end{array}\right] R_{k}\left[\begin{array}{c}
0 \\
\hat{K}_{k} D_{e k}
\end{array}\right]^{T} \\
& =\left(1-\beta_{k}\right) \beta_{k}\left[\begin{array}{c}
0 \\
\hat{K}_{k} D_{e k}
\end{array}\right] R_{k}\left[\begin{array}{c}
0 \\
\hat{K}_{k} D_{e k}
\end{array}\right]^{T} \\
& =\delta_{k}\left[\begin{array}{c}
0 \\
\hat{K}_{k} D_{e k}
\end{array}\right] R_{k}\left[\begin{array}{c}
0 \\
\hat{K}_{k} D_{e k}
\end{array}\right]^{T}
\end{aligned}
$$

with

$$
\delta_{k}=\left(1-\beta_{k}\right) \beta_{k} .
$$

It is noted that the deterministic uncertainty $F_{k}$ appears in (31). Therefore, it is impossible to have the exact value of the covariance matrix $\tilde{\Sigma}_{k}$. An alternative approach is to find a set of upper bounds for $\tilde{\Sigma}_{k}$ and then obtain the minimum with respect to filter parameters $\hat{A}_{k}$ and $\hat{K}_{k}$.

Before giving the upper bound, we present two lemmas.

Lemma 1: [28] Assume matrices $A, H, E$, and $F$ with compatible dimensions such that $F F^{T} \leq I$. Let $X$ be a symmetric positive definite matrix and let $\alpha>0$ be an arbitrary positive constant such that $\alpha^{-1} I-E X E^{T}>0$; then, the following inequality holds:

$$
\begin{aligned}
(A+H F E) X(A+H F E)^{T} \\
\leq A\left(X^{-1}-\alpha E^{T} E\right)^{-1} A^{T}+\alpha^{-1} H H^{T} .
\end{aligned}
$$

Lemma 2: [21] For $0 \leq k \leq N$, suppose $X=X^{T}>0$ and $s_{k}(X)=s_{k}^{T}(X) \in \mathbb{R}^{n \times \bar{n}}, h_{k}(X)=h_{k}^{T}(X) \in \mathbb{R}^{n \times n}$. If there exists $Y=Y^{T}>X$ such that

$$
\begin{aligned}
& s_{k}(Y) \geq s_{k}(X) \\
& h_{k}(Y) \geq s_{k}(Y)
\end{aligned}
$$

then applying solutions $M_{k}$ and $N_{k}$ to the following difference equations:

$$
M_{k+1}=s_{k}\left(M_{k}\right), N_{k+1}=h_{k}\left(N_{k}\right), M_{0}=N_{0}>0
$$

satisfies $M_{k} \leq N_{k}$.

The following corollary can be obtained immediately from Lemma 1 and (31), which provides a matrix recursive inequality for computing the actual covariance.

Corollary 1: If there exists an $\alpha_{k}$ such that $\alpha_{k}^{-1} I-$ $\tilde{E}_{k} \tilde{\Sigma}_{k} \tilde{E}_{k}^{T}>0$, the following inequality:

$$
\begin{aligned}
\tilde{\Sigma}_{k+1} \leq \tilde{A}_{k}\left(\tilde{\Sigma}_{k}^{-1}-\alpha_{k} \tilde{E}_{k}^{T} \tilde{E}_{k}\right)^{-1} \tilde{A}_{k}^{T}+\alpha_{k}^{-1} \tilde{H}_{k} \tilde{H}_{k}^{T} \\
+\tilde{B}_{1 k} Q_{k} \tilde{B}_{1 k}^{T}+\tilde{B}_{2 k} R_{k} \tilde{B}_{2 k}^{T}+\Psi_{k}+\Phi_{k}
\end{aligned}
$$

holds from (31).

Corollary 1 has "eliminated" the uncertainty $F_{k}$ in matrix (31). In order to design the quadratic filter associated with a positive definite matrix satisfying a Riccati-like inequality [28], we proceed to propose the notion of an "identity quadratic filter" for uncertain system (25). This "identity" is associated with a sequence of positive definite matrices satisfying a Riccati-like equation for all $\hat{A}_{k}$ and $\hat{K}_{k}$.

Definition 1: [27] Filter (22) is said to be an identity quadratic filter associated with a sequence of matrices $\Sigma_{k}=\Sigma_{k}^{T} \geq 0(0 \leq$ $k \leq N)$ if, for some positive scalars $\alpha_{k}(0 \leq k \leq N)$, the sequence $\Sigma_{k}$ satisfies

$$
\begin{aligned}
\Sigma_{k+1}=\tilde{A}_{k}\left(\Sigma_{k}^{-1}-\alpha_{k} \tilde{E}_{k}^{T} \tilde{E}_{k}\right)^{-1} \tilde{A}_{k}^{T}+\alpha_{k}^{-1} \tilde{H}_{k} \tilde{H}_{k}^{T} \\
\quad+\tilde{B}_{1 k} Q_{k} \tilde{B}_{1 k}^{T}+\tilde{B}_{2 k} R_{k} \tilde{B}_{2 k}^{T}+\Psi_{k}+\Phi_{k}
\end{aligned}
$$

and

$$
\alpha_{k}^{-1} I-\tilde{E}_{k} \Sigma_{k} \tilde{E}_{k}^{T}>0 .
$$

Remark 3: In this paper, our primary objective is to find an upper bound for the state estimation error variance and then minimize it. It will be shown later that, if we could design an identity quadratic filter of the form (22), there would exist positive definite solutions $\Sigma_{k}$ to (40) and (41) such that $\Sigma_{k}$ is an expected upper bound. Hence, it is important to investigate the existence as well as the algorithm for the solution to recursive matrix (40).

Based on Definition 1 and Lemma 2, we have the following conclusion that shows that solution $\Sigma_{k}$ to (40)-(41) indeed provides an upper bound for error covariance matrix $\tilde{\Sigma}_{k}$ in (31).

Theorem 1: Assume $\tilde{\Sigma}_{k}$ and $\Sigma_{k}$ satisfying (31) and (40)-(41), respectively. If $\Sigma_{0}=\tilde{\Sigma}_{0}$, then we have

$$
\tilde{\Sigma}_{k} \leq \Sigma_{k} \text {. }
$$

Proof: From (31), we denote

$$
\tilde{\Sigma}_{k+1}=s_{k}\left(\tilde{\Sigma}_{k}\right)
$$

where

$$
\begin{aligned}
s_{k}\left(\tilde{\Sigma}_{k}\right)= & \left(\tilde{A}_{k}+\tilde{H}_{k} F_{k} \tilde{E}_{k}\right) \tilde{\Sigma}_{k}\left(\tilde{A}_{k}+\tilde{H}_{k} F_{k} \tilde{E}_{k}\right)^{T} \\
& +\tilde{B}_{1 k} Q_{k} \tilde{B}_{1 k}^{T}+\tilde{B}_{2 k} R_{k} \tilde{B}_{2 k}^{T}+\Psi_{k}+\Phi_{k} .
\end{aligned}
$$

Denote also from (40) that

$$
\Sigma_{k+1}=h_{k}\left(\Sigma_{k}\right)
$$

where

$$
\begin{aligned}
h_{k}\left(\Sigma_{k}\right)= & \tilde{A}_{k}\left(\Sigma_{k}^{-1}-\alpha_{k} \tilde{E}_{k}^{T} \tilde{E}_{k}\right)^{-1} \tilde{A}_{k}^{T}+\alpha_{k}^{-1} \tilde{H}_{k} \tilde{H}_{k}^{T} \\
& +\tilde{B}_{1 k} Q_{k} \tilde{B}_{1 k}^{T}+\tilde{B}_{2 k} R_{k} \tilde{B}_{2 k}^{T}+\Psi_{k}+\Phi_{k} .
\end{aligned}
$$

It can be checked that functionals $h$ and $s$ defined above satisfy the conditions in Lemma 2 , hence the conclusion $\tilde{\Sigma}_{k} \leq \Sigma_{k}$.

Furthermore, in light of Definition 1 and Theorem 1, we have the following corollary.

Corollary 2: The inequality holds

$$
\begin{aligned}
\mathbb{E}\left[\left(x_{k}-\hat{x}_{k}\right)\left(x_{k}-\hat{x}_{k}\right)^{T}\right] & =\left[\begin{array}{ll}
I & -I
\end{array}\right] \tilde{\Sigma}_{k}\left[\begin{array}{ll}
I & -I
\end{array}\right]^{T} \\
& \leq\left[\begin{array}{ll}
I & -I
\end{array}\right] \Sigma_{k}\left[\begin{array}{ll}
I & -I
\end{array}\right]^{T}, \forall k .
\end{aligned}
$$

From Theorem 1 and Corollary 2, it is clear that, if (40) has symmetric positive definite solutions $\Sigma_{k}$ such that $\alpha_{k}^{-1} I-$ $\tilde{E}_{k} \Sigma_{k} \tilde{E}_{k}^{T}>0$, then the upper bound for the state estimation error variance can be obtained as $\Sigma_{k}$. Such solutions are of 
course not unique in general. In the next section, we will try to solve (40) while selecting filter parameters $\hat{A}_{k}$ and $\hat{K}_{k}$ such that the obtained upper bound is minimized.

\section{Finite-Horizon Suboptimal FiLter DEsign}

Here, we will design the filter based on the upper bound for state estimation error variance. First, we will provide sufficient conditions for existence of the identity quadratic filter (22) to satisfy constraints for the upper bound of the actual state estimation error variance. Second, we will design the filter that optimizes the upper bound of the actual state estimation error variance.

An identity quadratic filter is found in the following theorem.

Theorem 2: Let $\alpha_{k}>0$ be a sequence of positive scalars. If the following two discrete-time Riccati-like difference equations:

$$
\begin{aligned}
\Theta_{k+1}= & -A_{k}\left(\Theta_{k}^{-1}-\alpha_{k} E_{k}^{T} E_{k}\right)^{-1} \bar{C}_{k}^{T} R_{1, k}^{-1} \bar{C}_{k} \\
& \cdot\left(\Theta_{k}^{-1}-\alpha_{k} E_{k}^{T} E_{k}\right)^{-1} A_{k}^{T} \\
& +A_{k}\left(\Theta_{k}^{-1}-\alpha_{k} E_{k}^{T} E_{k}\right)^{-1} A_{k}^{T} \\
& +\alpha_{k}^{-1} H_{1, k} H_{1, k}^{T}+B_{k} Q_{k} B_{k}^{T}, \quad \Theta_{0}=S_{1} \\
P_{k+1}= & A_{k}\left(P_{k}^{-1}-\alpha_{k} E_{k}^{T} E_{k}\right)^{-1} A_{k}^{T}+\alpha_{k}^{-1} H_{1, k} H_{1, k}^{T} \\
& +B_{k} Q_{k} B_{k}^{T} \\
P_{0}= & S_{0} \geq S_{1}
\end{aligned}
$$

have positive-definite solutions $\Theta_{k}$ and $P_{k}$ such that

$$
\alpha_{k}^{-1} I-E_{k} P_{k} E_{k}^{T}>0
$$

then there exists an identity quadratic filter (22) with parameters

$$
\begin{aligned}
& \hat{A}_{k}=A_{k}+\left(A_{k}-\hat{K}_{k} \bar{C}_{k}\right) \Theta_{k} E_{k}^{T}\left(\alpha_{k}^{-1} I-E_{k} \Theta_{k} E_{k}^{T}\right)^{-1} E_{k} \\
& \hat{K}_{k}=A_{k}\left(\Theta_{k}^{-1}-\alpha_{k} E_{k}^{T} E_{k}\right)^{-1} \bar{C}_{k}^{T} R_{1, k}^{-1}
\end{aligned}
$$

where

$$
\begin{aligned}
R_{1, k}=\bar{D}_{k} R_{k} \bar{D}_{k}^{T}+\delta_{k} D_{e k} R_{k} D_{e k}^{T}+\delta_{k} C_{e k} P_{k} C_{e k}^{T} \\
+\bar{C}_{k}\left(\Theta_{k}^{-1}-\alpha_{k} E_{k}^{T} E_{k}\right)^{-1} \bar{C}_{k}^{T}
\end{aligned}
$$

such that the state estimation error variance satisfies the boundedness condition

$$
\mathbb{E}\left[\left(x_{k}-\hat{x}_{k}\right)\left(x_{k}-\hat{x}_{k}\right)^{T}\right] \leq \Theta_{k}, \quad \forall k .
$$

Proof: First, we need to find a solution to (40). Suppose that $\Sigma_{k}$ is of the form

$$
\Sigma_{k}=\left[\begin{array}{cc}
P_{k} & P_{k}-\Theta_{k} \\
P_{k}-\Theta_{k} & P_{k}-\Theta_{k}
\end{array}\right]
$$

where $\Theta_{k}$ and $P_{k}$ are defined in (44) and (45), respectively.

To prove $\Sigma_{k} \geq 0$ according to (44) and (45), we define

$$
\begin{aligned}
h\left(P_{k}\right)= & A_{k}\left(P_{k}^{-1}-\alpha_{k} E_{k}^{T} E_{k}\right)^{-1} A_{k}^{T} \\
& +\alpha_{k}^{-1} H_{1, k} H_{1, k}^{T}+B_{k} Q_{k} B_{k}^{T}
\end{aligned}
$$

$$
\begin{aligned}
s\left(\Theta_{k}\right)= & -A_{k}\left(\Theta_{k}^{-1}-\alpha_{k} E_{k}^{T} E_{k}\right)^{-1} \bar{C}_{k}^{T} R_{1, k}^{-1} \bar{C}_{k} \\
& \cdot\left(\Theta_{k}^{-1}-\alpha_{k} E_{k}^{T} E_{k}\right)^{-1} A_{k}^{T} \\
& +A_{k}\left(\Theta_{k}^{-1}-\alpha_{k} E_{k}^{T} E_{k}\right)^{-1} A_{k}^{T} \\
& +\alpha_{k}^{-1} H_{1, k} H_{1, k}^{T}+B_{k} Q_{k} B_{k}^{T} .
\end{aligned}
$$

Functionals $h$ and $s$ satisfy the conditions in Lemma 2, and thus it follows that $P_{k}-\Theta_{k} \geq 0$. This concludes that $\Sigma_{k} \geq 0$ in (51) from condition $P_{k}>0$. Also, from (46), we can obtain

$$
\alpha_{k}^{-1} I-\tilde{E}_{k} \Sigma_{k} \tilde{E}_{k}^{T}=\alpha_{k}^{-1} I-E_{k} P_{k} E_{k}^{T}>0
$$

which satisfies condition (41). It can be inferred that $\alpha_{k}^{-1} I-$ $E_{k} \Theta_{k} E_{k}^{T}=\alpha_{k}^{-1} I-E_{k} P_{k} E_{k}^{T}+E_{k}\left(P_{k}-\Theta_{k}\right) E_{k}^{T}>0$.

It remains to be shown that (51) is a solution to (40). Substituting filter parameter expressions (47), (48), and (51) into the right-hand side of (40) and considering conditions (44) and (45), straightforward algebraic manipulations show that the right-hand side of (40) is given by

$$
\begin{aligned}
& \tilde{A}_{k}\left(\Sigma_{k}^{-1}-\alpha_{k} \tilde{E}_{k}^{T} \tilde{E}_{k}\right)^{-1} \tilde{A}_{k}^{T}+\alpha_{k}^{-1} \tilde{H}_{k} \tilde{H}_{k}^{T} \\
& \quad+\tilde{B}_{1 k} Q_{k} \tilde{B}_{1 k}^{T}+\tilde{B}_{2 k} R_{k} \tilde{B}_{2 k}^{T}+\Psi_{k}+\Phi_{k} \\
& =\left[\begin{array}{cc}
P_{k+1} & P_{k+1}-\Theta_{k+1} \\
P_{k+1}-\Theta_{k+1} & P_{k+1}-\Theta_{k+1}
\end{array}\right] .
\end{aligned}
$$

This means that (51) is a solution to (40). Also, from Corollary 2 , we can conclude that

$\mathbb{E}\left[\left(x_{k}-\hat{x}_{k}\right)\left(x_{k}-\hat{x}_{k}\right)^{T}\right] \leq\left[\begin{array}{ll}I & -I\end{array}\right] \Sigma_{k}\left[\begin{array}{ll}I & -I\end{array}\right]^{T}=\Theta_{k}, \quad \forall k$.

In the following theorem, we will prove that filter (22) with parameters (47) and (48) is optimal.

Theorem 3: If (44) and (45) have positive-definite solutions $\Theta_{k}$ and $P_{k}$ such that $\alpha_{k}^{-1} I-E_{k} P_{k} E_{k}^{T}>0$, then identity quadratic filter (22) with parameters (47) and (48) minimizes bound $\Theta_{k}$.

Proof: We prove that the filter's parameters given in (47) and (48) are optimal in the sense that they optimize upper bound $\Theta_{k+1}$. From (27), (29), (40), and (51), we have

$$
\begin{aligned}
\Theta_{k+1}= & {\left[\begin{array}{ll}
I & -I
\end{array}\right] \Sigma_{k+1}\left[\begin{array}{ll}
I & -I
\end{array}\right]^{T} } \\
= & \alpha_{k}^{-1} H_{1, k} H_{1, k}^{T}+B_{k} Q_{k} B_{k}^{T}+\hat{K}_{k} \bar{D}_{k} R_{k} \bar{D}_{k}^{T} \hat{K}_{k}^{T} \\
& +\delta_{k} \hat{K}_{k} D_{e k} R_{k} D_{e k}^{T} \hat{K}_{k}^{T}+\delta_{k} \hat{K}_{k} C_{e k} P_{k} C_{e k}^{T} \hat{K}_{k}^{T} \\
& +\left[\begin{array}{ll}
A_{k}-\hat{K}_{k} \bar{C}_{k} & \hat{K}_{k} \bar{C}_{k}-\hat{A}_{k}
\end{array}\right] \\
& \cdot\left(\Sigma_{k}^{-1}-\alpha_{k} \tilde{E}_{k}^{T} \tilde{E}_{k}\right)^{-1} \\
& \cdot\left[\begin{array}{ll}
A_{k}-\hat{K}_{k} \bar{C}_{k} & \hat{K}_{k} \bar{C}_{k}-\hat{A}_{k}
\end{array}\right]^{T} .
\end{aligned}
$$

Obviously, $\Theta_{k+1}$ is dependent on the parameters $\hat{A}_{k}$ and $\hat{K}_{k}$. In order to determine optimal filter parameters $\hat{A}_{k}$ and $\hat{K}_{k}$ that minimize $\Theta_{k+1}$, we take the first variation to (57) and obtain

$$
\begin{aligned}
2\left[A_{k}-\hat{K}_{k} \bar{C}_{k} \quad \hat{K}_{k} \bar{C}_{k}-\hat{A}_{k}\right] & \\
& \cdot\left(\Sigma_{k}^{-1}-\alpha_{k} \tilde{E}_{k}^{T} \tilde{E}_{k}\right)^{-1}\left[\begin{array}{ll}
0 & -I
\end{array}\right]^{T}=0
\end{aligned}
$$


and

$$
\begin{aligned}
& 2 \hat{K}_{k} \bar{D}_{k} R_{k} \bar{D}_{k}^{T}+2 \delta_{k} \hat{K}_{k} D_{e k} R_{k} D_{e k}^{T}+2 \delta_{k} \hat{K}_{k} C_{e k} P_{k} C_{e k}^{T} \\
& +2\left[A_{k}-\hat{K}_{k} \bar{C}_{k}\right. \\
& \left.\quad \hat{K}_{k} \bar{C}_{k}-\hat{A}_{k}\right] \\
& \cdot\left(\Sigma_{k}^{-1}-\alpha_{k} \tilde{E}_{k}^{T} \tilde{E}_{k}\right)^{-1}\left[\begin{array}{ll}
-\bar{C}_{k} & \bar{C}_{k}
\end{array}\right]^{T}=0
\end{aligned}
$$

From (58) and facts

$$
\begin{aligned}
& {\left[\begin{array}{ll}
I & -I
\end{array}\right]\left(\Sigma_{k}^{-1}-\alpha_{k} \tilde{E}_{k}^{T} \tilde{E}_{k}\right)^{-1}\left[\begin{array}{ll}
0 & -I
\end{array}\right]^{T}} \\
& =\Theta_{k} E_{k}^{T}\left(\alpha_{k}^{-1} I-E_{k} P_{k} E_{k}^{T}\right)^{-1} E_{k}\left(\Theta_{k}-P_{k}\right) \\
& {\left[\begin{array}{ll}
0 & I
\end{array}\right]\left(\Sigma_{k}^{-1}-\alpha_{k} \tilde{E}_{k}^{T} \tilde{E}_{k}\right)^{-1}\left[\begin{array}{ll}
0 & -I
\end{array}\right]^{T}} \\
& =\left(\Theta_{k}-P_{k}\right)-\left(\Theta_{k}-P_{k}\right) E_{k}^{T}\left(\alpha_{k}^{-1} I-E_{k} P_{k} E_{k}^{T}\right)^{-1} \\
& \quad \cdot E_{k}\left(\Theta_{k}-P_{k}\right)
\end{aligned}
$$

we can determine optimal filter parameter $\hat{A}_{k}$ as

$$
\begin{aligned}
\hat{A}_{k}= & A_{k}+\left(A_{k}-\hat{K}_{k} \bar{C}_{k}\right) \Theta_{k} E_{k}^{T}\left(\alpha_{k}^{-1} I-E_{k} P_{k} E_{k}^{T}\right)^{-1} \\
& \cdot E_{k}\left[I-\left(\Theta_{k}-P_{k}\right) E_{k}^{T}\left(\alpha_{k}^{-1} I-E_{k} P_{k} E_{k}^{T}\right)^{-1} E_{k}\right]^{-1} \\
= & A_{k}+\left(A_{k}-\hat{K}_{k} \bar{C}_{k}\right) \Theta_{k} E_{k}^{T} E_{k}\left(\alpha_{k}^{-1} I-P_{k} E_{k}^{T} E_{k}\right)^{-1} \\
& \cdot\left[I-\left(\Theta_{k}-P_{k}\right) E_{k}^{T} E_{k}\left(\alpha_{k}^{-1} I-P_{k} E_{k}^{T} E_{k}\right)^{-1}\right]^{-1} \\
= & A_{k}+\left(A_{k}-\hat{K}_{k} \bar{C}_{k}\right) \Theta_{k} E_{k}^{T} E_{k}\left(\alpha_{k}^{-1} I-\Theta_{k} E_{k}^{T} E_{k}\right)^{-1} \\
= & A_{k}+\left(A_{k}-\hat{K}_{k} \bar{C}_{k}\right) \Theta_{k} E_{k}^{T}\left(\alpha_{k}^{-1} I-E_{k} \Theta_{k} E_{k}^{T}\right)^{-1} E_{k}
\end{aligned}
$$

which is identical to (47).

Our next task is to derive optimal filter parameter $\hat{K}_{k}$ from (59) and show that it is actually (48). By using

$$
\begin{aligned}
& {\left[\begin{array}{ll}
I & -I
\end{array}\right]\left(\Sigma_{k}^{-1}-\alpha_{k} \tilde{E}_{k}^{T} \tilde{E}_{k}\right)^{-1}\left[\begin{array}{ll}
I & -I
\end{array}\right]^{T}} \\
& \quad=\Theta_{k}+\Theta_{k} E_{k}^{T}\left(\alpha_{k}^{-1} I-E_{k} P_{k} E_{k}^{T}\right)^{-1} E_{k} \Theta_{k} \\
& {\left[\begin{array}{ll}
0 & -I
\end{array}\right]\left(\Sigma_{k}^{-1}-\alpha_{k} \tilde{E}_{k}^{T} \tilde{E}_{k}\right)^{-1}\left[\begin{array}{ll}
I & -I
\end{array}\right]^{T}} \\
& \quad=\left(\Theta_{k}-P_{k}\right) E_{k}^{T}\left(\alpha_{k}^{-1} I-E_{k} P_{k} E_{k}^{T}\right)^{-1} E_{k} \Theta_{k}
\end{aligned}
$$

we have

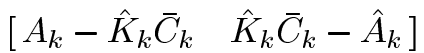

$$
\begin{aligned}
& \cdot\left(\Sigma_{k}^{-1}-\alpha_{k} \tilde{E}_{k}^{T} \tilde{E}_{k}\right)^{-1}\left[\begin{array}{ll}
I & -I
\end{array}\right]^{T} \\
& =\left(A_{k}-\hat{K}_{k} \bar{C}_{k}\right)\left[\Theta_{k}+\Theta_{k} E_{k}^{T}\left(\alpha_{k}^{-1} I-E_{k} P_{k} E_{k}^{T}\right)^{-1}\right. \\
& \text { - } \left.E_{k} \Theta_{k}\right]-\left(A_{k}-\hat{A}_{k}\right)\left(\Theta_{k}-P_{k}\right) E_{k}^{T} \\
& \text { · }\left(\alpha_{k}^{-1} I-E_{k} P_{k} E_{k}^{T}\right)^{-1} E_{k} \Theta_{k} \\
& =\left(A_{k}-\hat{K}_{k} \bar{C}_{k}\right)\left[\Theta_{k}+\Theta_{k} E_{k}^{T}\left(\alpha_{k}^{-1} I-E_{k} P_{k} E_{k}^{T}\right)^{-1}\right. \\
& \text { - } \left.E_{k} \Theta_{k}\right]+\left(A_{k}-\hat{K}_{k} \bar{C}_{k}\right) \Theta_{k} E_{k}^{T} E_{k} \\
& \text { - }\left(\alpha_{k}^{-1} I-\Theta_{k} E_{k}^{T} E_{k}\right)^{-1}\left(\Theta_{k}-P_{k}\right) \\
& \text { - } E_{k}^{T}\left(\alpha_{k}^{-1} I-E_{k} P_{k} E_{k}^{T}\right)^{-1} E_{k} \Theta_{k} \\
& =\left(A_{k}-\hat{K}_{k} \bar{C}_{k}\right)\left[\Theta_{k}+\Theta_{k} E_{k}^{T}\left(\alpha_{k}^{-1} I-E_{k} P_{k} E_{k}^{T}\right)^{-1}\right. \\
& \text { - } \left.E_{k} \Theta_{k}\right]-\left(A_{k}-\hat{K}_{k} \bar{C}_{k}\right)\left[\left(\alpha_{k}^{-1} I-\Theta_{k} E_{k}^{T} E_{k}\right)\right. \\
& \text { - } \left.\alpha_{k}^{-1} I\right]\left(\alpha_{k}^{-1} I-\Theta_{k} E_{k}^{T} E_{k}\right)^{-1}\left(\Theta_{k}-P_{k}\right) \\
& \text { - } E_{k}^{T}\left(\alpha_{k}^{-1} I-E_{k} P_{k} E_{k}^{T}\right)^{-1} E_{k} \Theta_{k} \\
& =\left(A_{k}-\hat{K}_{k} \bar{C}_{k}\right) \Theta_{k}+\left(A_{k}-\hat{K}_{k} \bar{C}_{k}\right) P_{k} E_{k}^{T} \\
& \cdot\left(\alpha_{k}^{-1} I-E_{k} P_{k} E_{k}^{T}\right)^{-1} E_{k} \Theta_{k} \\
& +\alpha_{k}^{-1}\left(A_{k}-\hat{K}_{k} \bar{C}_{k}\right)\left(\alpha_{k}^{-1} I-\Theta_{k} E_{k}^{T} E_{k}\right)^{-1}
\end{aligned}
$$

$$
\begin{aligned}
& \cdot\left(\Theta_{k}-P_{k}\right) E_{k}^{T}\left(\alpha_{k}^{-1} I-E_{k} P_{k} E_{k}^{T}\right)^{-1} E_{k} \Theta_{k} \\
= & \left(A_{k}-\hat{K}_{k} \bar{C}_{k}\right)\left\{I+\left[P_{k}+\left(I-\alpha_{k} \Theta_{k} E_{k}^{T} E_{k}\right)^{-1}\right.\right. \\
& \left.\left.\cdot\left(\Theta_{k}-P_{k}\right)\right] E_{k}^{T}\left(\alpha_{k}^{-1} I-E_{k} P_{k} E_{k}^{T}\right)^{-1} E_{k}\right\} \Theta_{k} \\
= & \left(A_{k}-\hat{K}_{k} \bar{C}_{k}\right)\left[I+\left(I-\alpha_{k} \Theta_{k} E_{k}^{T} E_{k}\right)^{-1}\right. \\
& \cdot\left(-\alpha_{k} \Theta_{k} E_{k}^{T} E_{k} P_{k}+\Theta_{k}\right)\left(\alpha_{k}^{-1} I-E_{k}^{T} E_{k} P_{k}\right)^{-1} \\
& \left.\cdot E_{k}^{T} E_{k}\right] \Theta_{k} \\
= & \left(A_{k}-\hat{K}_{k} \bar{C}_{k}\right)\left[I+\left(\alpha_{k}^{-1} I-\Theta_{k} E_{k}^{T} E_{k}\right)^{-1}\right. \\
& \left.\cdot \Theta_{k} E_{k}^{T} E_{k}\right] \Theta_{k} \\
= & \left(A_{k}-\hat{K}_{k} \bar{C}_{k}\right)\left(\Theta_{k}^{-1}-\alpha_{k} E_{k}^{T} E_{k}\right)^{-1} .
\end{aligned}
$$

Substituting (60) into (59), optimal filter parameter $\hat{K}_{k}$ is given by

$$
\hat{K}_{k}=A_{k}\left(\Theta_{k}^{-1}-\alpha_{k} E_{k}^{T} E_{k}\right)^{-1} \bar{C}_{k}^{T} R_{1, k}^{-1}
$$

where

$$
\begin{aligned}
R_{1, k}=\bar{D}_{k} R_{k} \bar{D}_{k}^{T}+\delta_{k} D_{e k} R_{k} D_{e k}^{T}+\delta_{k} C_{e k} P_{k} C_{e k}^{T} \\
+\bar{C}_{k}\left(\Theta_{k}^{-1}-\alpha_{k} E_{k}^{T} E_{k}\right)^{-1} \bar{C}_{k}^{T} .
\end{aligned}
$$

Note that (61) is identical to (48). It becomes clear now that filter parameters given in (47) and (48) are indeed optimal and minimize the upper-bound $\Theta_{k}$ for the actual estimation error variance.

Remark 4: Theorems 2 and 3 provide the optimal filter design by optimizing the upper bound for state estimation error variance. One-step-ahead variance bound is optimized by selecting the filter parameters $\hat{A}_{k}$ and $\hat{K}_{k}$ as in (47) and (48) under given scaling parameter $\alpha_{k}$. The optimization is step by step by solving the Riccati-like difference (44) and (45). Since the $n$-dimensional system with randomly varying sensor delays is converted into a stochastic parameter system with dimension of $2 n$, two $2 n$-dimensional recursive Riccati-like difference (44) and (45) will be computed in the filter design algorithm.

Remark 5: Note that the Riccati-like difference (44) and (45) involve scalar parameter $\alpha_{k}$. Detailed discussions on the feasibility and convergent properties of such kind of Riccati-like difference equations can be found in [32]. A simple tuning timevarying scaling parameter $\alpha_{k}$ has been realized for variance constraint in [11]. As seen from (44)-(46), a smaller $\alpha_{k}$ would make it easier for (44) and (45) to have positive-definite solutions and the positive-definite condition in (46) is easier to be satisfied. As a cost, the upper bounds could be less tighter. Notice that a larger $\alpha_{k}$ could lead to the possibility that (44) and (45) have no positive-definite solutions and the positive-definite condition in (46) is not satisfied. Such a phenomenon is confirmed in the example of Section V. Therefore, it is important to choose an appropriate $\alpha_{k}$. Note that a semi-definite programming approach to optimizing multiple scaling parameters has been proposed in [5] where thorough studies have been conducted on how the scaling parameters affect estimation performance. Finally, we point out that although there have been several algorithms available for tuning time-varying scaling parameter $\alpha_{k}$ in [5], [11], [32] the question of how to correctly utilize the scenario with respect to these parameters is still an open problem deserving further study. 


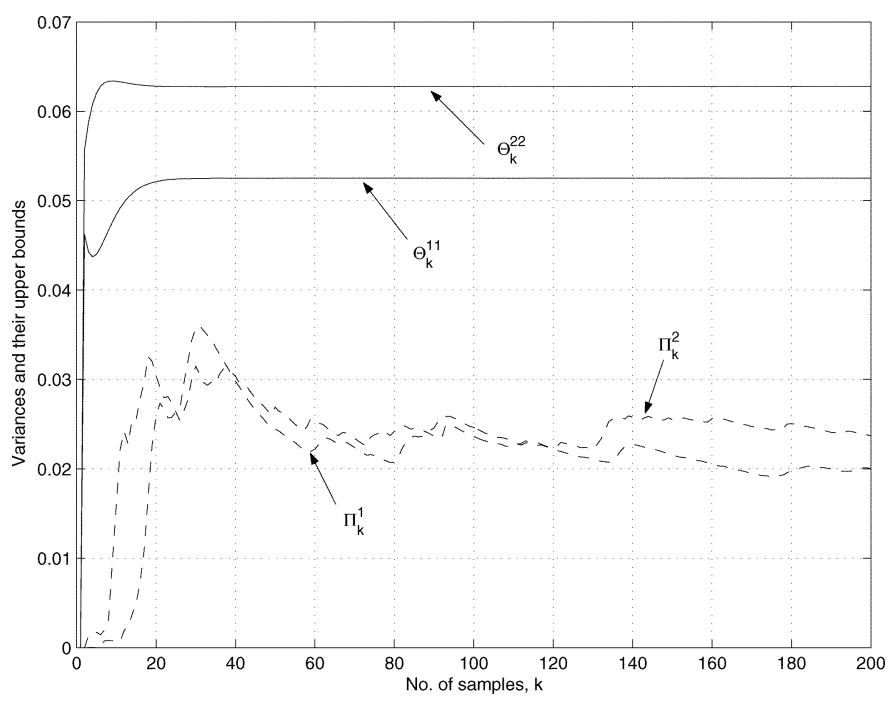

Fig. 1. Actual variances $\Pi_{k}^{1}$ and $\Pi_{k}^{2}$ as well as their upper bounds $\Theta_{k}^{11}$ and $\Theta_{k}^{22}$ in the case of $\alpha_{k}=2$ and $\beta_{k}=0.95$.

\section{NUMERICAL EXAMPLE}

Consider a target tracking system

$$
\left\{\begin{array}{l}
\vec{x}_{k+1}=\left(\left[\begin{array}{cc}
0.9 & T \\
0 & 0.9
\end{array}\right]+\left[\begin{array}{l}
0.1 \\
0.1
\end{array}\right] F_{k}\left[\begin{array}{ll}
0.2 & 0.2
\end{array}\right]\right) \vec{x}_{k}+\left[\begin{array}{c}
\frac{T^{2}}{2} \\
T
\end{array}\right] w_{k} \\
\vec{y}_{k}=\left[\begin{array}{ll}
0.5 & 1
\end{array}\right] \vec{x}_{k}+\vec{v}_{k}
\end{array}\right.
$$

where $T$ is the sample period, the state $\vec{x}_{k}=[s(k) \quad \dot{s}(k)]^{T}$ is composed of the position and the velocity of the target at time $k T, \vec{y}_{k}$ is the measured output, $F_{k}$ is a deterministic perturbation matrix satisfying $F_{k} F_{k}^{T} \leq I$, and $w_{k}$ and $\vec{v}_{k}$ are mutually independent zero-mean Gaussian white noise sequences with unity covariances.

In order to estimate the position and velocity of the tracked target, measured output $\vec{y}_{k}$ is sent to the monitoring center via network-based communication. Due to the limited bandwidth of the communication channel, the measured output arrives with the following random transmission delay

$$
y_{k}=\left(1-\gamma_{k}\right) \vec{y}_{k}+\gamma_{k} \vec{y}_{k-1}
$$

where stochastic variable $\gamma_{k} \in \mathbb{R}$ is a Bernoulli distributed white sequence taking values on 0 or 1 with $\operatorname{Prob}\left\{\gamma_{k}=1\right\}=$ $\mathbb{E}\left\{\gamma_{k}\right\}=0.95$.

In the simulation, sample period $T$ is chosen as $0.1 \mathrm{~s}$ and $F_{k}$ as $\sin (0.6 k)$. Initial values are set as $\vec{x}_{0}=\left[\begin{array}{ll}1 & 0\end{array}\right]^{T}, S_{0}=$ $0.1 I_{4}$, and $S_{1}=0.05 I_{4}$. The simulation results are obtained by solving (44) and (45) in Theorem 2 with parameters $\alpha_{k}$ and $\beta_{k}$. The plots of upper bounds $\Theta_{k}^{11}$ and $\Theta_{k}^{22}$ (solid lines) as well as the actual variances for the states $\Pi_{k}^{1}=\operatorname{Cov}\left(x_{k}^{1}-\hat{x}_{k}^{1}\right)$ and $\Pi_{k}^{2}=\operatorname{Cov}\left(x_{k}^{2}-\hat{x}_{k}^{2}\right)$ (dashed lines) are given in Figs. 1-3. It can be seen from Figs. 1 and 2 that the upper bounds become tighter as $\alpha_{k}$ increases, but there could be no solutions to (44) and (45) if $\alpha_{k}$ is too large. Therefore, the best choice for $\alpha_{k}$ is to make it as large as possible providing (44) and (45) are feasible. In addition, the case of misestimating $\beta_{k}$ is investigated in Fig. 3. When $\beta_{k}=0.9$ and we misestimate it as 0.7 , the

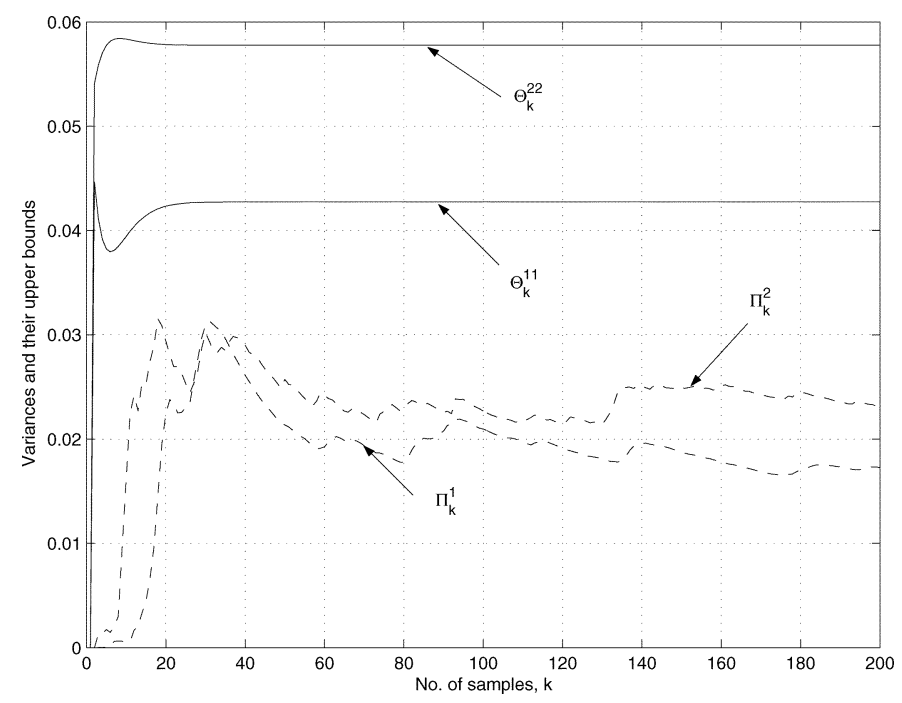

Fig. 2. Actual variances $\Pi_{k}^{1}$ and $\Pi_{k}^{2}$ as well as their upper bounds $\Theta_{k}^{11}$ and $\Theta_{k}^{22}$ in the case of $\alpha_{k}=3$ and $\beta_{k}=0.95$.

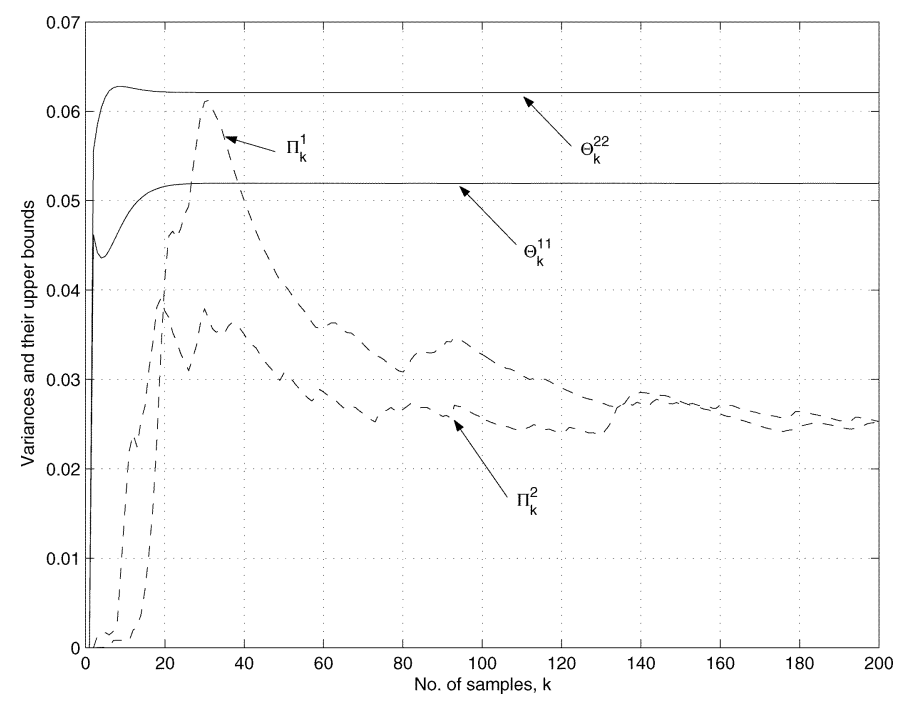

Fig. 3. Actual variances $\Pi_{k}^{1}$ and $\Pi_{k}^{2}$ as well as their upper bounds $\Theta_{k}^{11}$ and $\Theta_{k}^{22}$ in the case of $\alpha_{k}=2$ and $\beta_{k}=0.9$ (misestimation of $\beta_{k}$ as 0.7 ).

requirements that the actual variances for the states stay below their upperbounds are not satisfied, which implies the proposed algorithm is sensitive to the misestimation of $\beta_{k}$.

\section{CONCLUSION}

In this paper, a new robust filtering problem with delayed sensor measurements has been considered for discrete timevarying systems subject to norm-bounded parameter uncertainties. An algorithm has been provided for designing a finitehorizon filter which guarantees an optimized upper bound on the state estimation error variance, for all stochastic sensor delays and admissible deterministic uncertainties. Simulation results demonstrate the feasibility of our algorithm. Our future research topics would include the design of a reduced-order filter within the same framework and the design of a nonlinear filter that first detects whether delays occur and then proceeds according to the detection. 


\section{REFERENCES}

[1] B. D. O. Anderson and J. B. Moore, Optimal Filtering. Englewood Cliffs, NJ: Prentice-Hall, 1979.

[2] X. Chen and K. Zhou, " $H_{\infty}$ Gaussian filter on infinite time horizon," IEEE Trans. Circuits Syst. I, Fundam. Theory Appl., vol. 49, no. 5, pp. 674-679, May 2002.

[3] W. L. DeKoning, "Optimal estimation of linear discrete time systems with stochastic parameters," Automatica, vol. 20, pp. 113-115, 1984.

[4] E. Fridman and U. Shaked, "A new $H_{\infty}$ filter design for linear time delay systems," IEEE Trans. Signal Process., vol. 49, no. 11, pp. 2839-2843, Nov. 2001.

[5] M. Fu, C. E. de Souza, and Z. Luo, "Finite horizon robust Kalman filter design,” IEEE Trans. Signal Process., vol. 49, no. 9, pp. 2103-2112, Sep. 2001.

[6] J. C. Geromel, "Optimal linear filtering under parameter uncertainty," IEEE Trans. Signal Process., vol. 47, no. 1, pp. 168-175, Jan. 1999.

[7] J. C. Geromel, M. C. de Oliveira, and J. Bernussou, "Robust filtering of discrete-time linear systems with parameter dependent Lyapunov functions," SIAM J. Control Optim., vol. 41, no. 3, pp. 700-711, 2002.

[8] H. Gao and C. Wang, "Robust $L_{2}-L_{\infty}$ filtering for uncertain systems with multiple time-varying state delays," IEEE Trans. Circuits Syst. I, Fundam. Theory Appl., vol. 50, no. 4, pp. 594-599, Apr. 2003.

[9] H. Gao and C. Wang, "A delay-dependent approach to robust $H_{\infty}$ filtering for uncertain discrete-time state-delayed systems," IEEE Trans. Signal Process., vol. 52, no. 6, pp. 1631-1640, Jun. 2004.

[10] H. Gao and C. Wang, "Delay-dependent robust $H_{\infty}$ and $\mathrm{H}$-infinity and $L_{2}-L_{\infty}$ filtering for a class of uncertain nonlinear time-delay systems," IEEE Trans. Autom. Control, vol. 48, no. 9, pp. 1661-1666, Sep. 2003.

[11] Y. S. Hung and F. Yang, "Robust $H_{\infty}$ filtering with error variance constraints for uncertain discrete time-varying systems with uncertainty," Automatica, vol. 39, no. 7, pp. 1185-1194, 2003.

[12] M. Mahmoud, Robust Control and Filtering for Time-Delay Systems. New York: Marcel Dekker, 2000.

[13] M. Malek-Zavarei and M. Jamshidi, Time-Delay Systems: Analysis, Optimization and Application. Amsterdam, The Netherlands: NorthHolland, 1987.

[14] G. Millerioux and J. X. Daafouz, "Global chaos synchronization and robust filtering in noisy context," IEEE Trans. Circuits Syst. I, Fundam. Theory Appl., vol. 48, no. 10, pp. 1170-1176, Oct. 2001.

[15] S. O. R. Moheimani, A. V. Savkin, and I. R. Petersen, "Robust filtering, prediction, smoothing, and observability of uncertain systems," IEEE Trans. Circuits Syst. I, Fundam. Theory Appl., vol. 45, no. 4, pp. 446-457, Apr. 1998

[16] R. M. Palhares, C. E. de Souza, and P. L. D. Peres, "Robust $H_{\infty}$ filtering for uncertain discrete-time state-delayed systems," IEEE Trans. Signal Process., vol. 49, no. 8, pp. 1696-1703, Aug. 2001.

[17] I. Petersen and A. V. Savkin, Robust Kalman Filtering for Signals and Systems with Large Uncertainties. Boston, MA: Birkhauser, 1999, Control Engineering.

[18] A. W. Pila, U. Shaked, and C. E. de Souza, " $H_{\infty}$ filtering for continuous linear system with delay," IEEE Trans. Autom. Control, vol. 44, no. 7, pp. 1412-1417, Jul. 1999.

[19] A. Ray, "Output feedback control under randomly varying distributed delays," J. Guid., Control Dynam., vol. 17, no. 4, pp. 701-711, Jul. 1994.

[20] U. Shaked, L. Xie, and Y. C. Soh, "New approaches to robust minimum variance filter design," IEEE Trans. Signal Process., vol. 49, no. 11, pp. 2620-2629, Nov. 2001.

[21] Y. Theodor and U. Shaked, "Robust discrete-time minimum-variance filtering," IEEE Trans. Signal Process., vol. 44, no. 2, pp. 181-189, Feb. 1996.

[22] F. Wang and V. Balakrishnan, "Robust Kalman filters for linear timevarying systems with stochastic parametric uncertainties," IEEE Trans. Signal Process., vol. 50, no. 4, pp. 803-813, Apr. 2002.

[23] Z. Wang, D. W. C. Ho, and X. Liu, "Robust filtering under randomly varying sensor delay with variance constraints," IEEE Trans. Circuits Syst. II, Exp. Briefs, vol. 51, no. 6, pp. 320-326, Jun. 2004

[24] Z. Wang and F. Yang, "Robust filtering for uncertain linear systems with delayed states and outputs," IEEE Trans. Circuits Syst. I, Fundam. Theory Appl., vol. 49, no. 1, pp. 125-130, Jan. 2002.

[25] Z. Wang and H. Unbehauen, "Robust $H_{2} / H_{\infty}$-state estimation for systems with error variance constraints: The continuous-time case," IEEE Trans. Autom. Control, vol. 44, no. 5, pp. 1061-1065, May 1999.

[26] Z. Wang, Z. Guo, and H. Unbehauen, "Robust $H_{2} / H_{\infty}$-state estimation for discrete-time systems with error variance constraints," IEEE Trans. Autom. Control, vol. 42, no. 10, pp. 1431-1435, Oct. 1997.
[27] L. Xie and Y. C. Soh, "Guaranteed cost control of uncertain discrete-time systems," Control - Theory Adv. Technol., vol. 10, no. 4, pp. 1235-1251, Aug. 1995

[28] L. Xie, Y. C. Soh, and C. E. de Souza, "Robust Kalman filtering for uncertain discrete-time systems," IEEE Trans. Autom. Control, vol. 39, no. 6, pp. 1310-1314, Jun. 1994

[29] F. Yang, Z. Wang, and Y. S. Hung, "Robust Kalman filtering for discrete time-varying uncertain systems with multiplicative noise," IEEE Trans. Autom. Control, vol. 47, no. 7, pp. 1179-1183, Jul. 2002.

[30] E. Yaz and A. Ray, "Linear unbiased state estimation for random models with sensor delay," in Proc. Conf. Decision \& Control, Kobe, Japan, Dec. 1996, pp. 47-52.

[31] K. S. Yeung and S. C. Chan, "The design and multiplier-less realization of software radio receivers with reduced system delay," IEEE Trans. Circuits Syst. I, Reg. Papers, vol. 51, no. 12, pp. 2444-2459, Dec. 2004.

[32] X. Zhu, Y. C. Soh, and L. Xie, "Design and analysis of discrete-time robust Kalman filters," Automatica, vol. 38, no. 6, pp. 1069-1077, Jun. 2002.

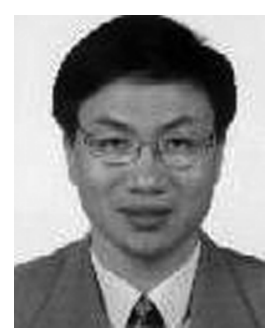

Fuwen Yang (SM'04) received the B.Sc. degree in electrical engineering from Anhui University of Science and Technology, Huainan, China, in 1983 and the M.Sc. and Ph.D. degrees in control engineering from Huazhong University of Science and Technology, Wuhan, China, in 1986 and 1990, respectively.

$\mathrm{He}$ is currently a Professor with the School of Information Science and Engineering, East China University of Science and Technology, Shanghai Before joining East China University of Science and Technology, he was a Professor with Fuzhou University. He also held research positions at King's College London, Brunel University, the University of Manchester, and the University of Hong Kong. His research interests include $H_{\infty}$ control and filtering, iterative learning control, nonfragile control, signal processing, industrial real-time control, and power electronics. He has published more than 90 journal and conference papers in the general area of control engineering and signal processing.

Dr. Yang was a recipient of the Teaching Excellence Award for Young Teachers in 1995 from Fok Ying Tung Education Foundation, China, three Science and Technology Development Awards in 1996, 1999, and 2002, from Fujian Province of China, two Youth Science and Technology Awards both in 1998 from Fujian Province of China and from Yun Sheng Foundation. He was listed as one of the Ten Outstanding Youths from Fujian Province of China in 1999 and was honored with the May 4 Youth Medal from Fujian Province of China in 2000. Since 2001, he has been acting as a State Consultant receiving Special Allowance from the State Council of the People's Republic of China.

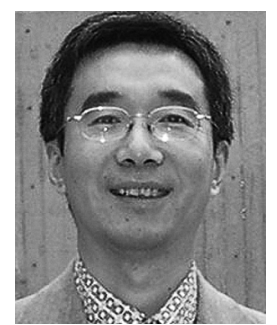

Zidong Wang (SM'03) was born in Jiangsu, China, in 1966 . He received the B.Sc. degree in mathematics from Suzhou University, Suzhou, China, in 1986 and the M.Sc. degree in applied mathematics and the $\mathrm{Ph} . \mathrm{D}$. degree in electrical and computer engineering from Nanjing University of Science and Technology, Nanjing, China, in 1990 and 1994, respectively.

He is now a Professor of Dynamical Systems and Computing with Brunel University, Middlesex, U.K. He was appointed as a Lecturer in 1990 and an Associate Professor in 1994 at Nanjing University of Science and Technology. From January 1997 to December 1998, he was an Alexander von Humboldt Research Fellow with the Control Engineering Laboratory, Ruhr-University Bochum, Germany. From January 1999 to February 2001, he was a Lecturer with the Department of Mathematics, University of Kaiserslautern, Germany. From March 2001 to July 2002, he was a University Senior Research Fellow with the School of Mathematical and Information Sciences, Coventry University, U.K. In August 2002, he joined the Department of Information Systems and Computing, Brunel University, as a Lecturer and was promoted to Reader in September 2003 and to Professor in July 2007. He also holds an Adjunct TePin Professorship at Donghua University, Shanghai, a Visiting Professorship at Fuzhou University, Fuzhou, and a Guest Professorship at Nanjing Normal University, Nanjing, China. His research interests include dynamical systems, signal processing, bioinformatics, and control theory and applications. He has published more than 120 papers in refereed international journals. 
Dr. Wang is a Fellow of the Royal Statistical Society, a member of program committee for many international conferences, and a very active reviewer for many international journals. He was awarded the Humboldt research fellowship in 1996 from Alexander von Humboldt Foundation, the JSPS Research Fellowship in 1998 from Japan Society for the Promotion of Science, and the William Mong Visiting Research Fellowship in 2002 from the University of Hong Kong. He was a recipient of the Outstanding Science and Technology Development Awards, once in 2005 and twice in 1997, from the National Education Committee of China, once in 1997 from the Military Industry General Company of China, once in 1997 from Jiangsu Province Government of China, and once in 1999 from the National Education Ministry of China. He is currently serving as an Associate Editor for IEEE TRANSACTIONS ON AUTOMATIC CONTROL, IEEE Transactions on NeUral Networks, IEEE Transactions on Signal PROCESSING, IEEE TRANSACTIONS ON SYSTEMS, MAN, AND Cybernetics - Part C, IEEE Transactions on Control Systems Technology, and Circuits, Systems \& Signal Processing, an Action Editor for Neural Networks, an Editorial Board Member for International Journal of Systems Science, Neurocomputing, International Journal of Computer Mathematics, International Journal of General Systems, and an Associate Editor on the Conference Editorial Board for the IEEE Control Systems Society. He was nominated an appreciated reviewer for IEEE TRANSACTIONS ON SIGNAL PROCESSING in 2006, an outstanding reviewer for IEEE TRANSACTIONS ON AUTOMATIC CONTROL in 2004 and for the journal Automatica in 2000. He received the Standing Membership of the Technical Committee on Control of IASTED (International Association of Science and Technology for Development) in 2000. From 2001 to 2008 he served as a member of technical program committee for 48 international conferences in the general area of systems theory and computing.

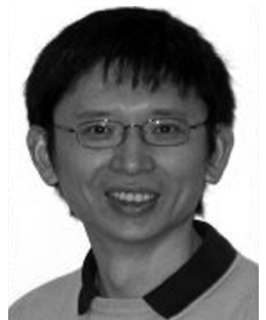

Gang Feng (SM'95-F'09) received the B.Eng and M.Eng. degrees in automatic control (of electrical engineering) from Nanjing Aeronautical Institute, Nanjing, China in 1982 and 1984, respectively, and the $\mathrm{Ph} . \mathrm{D}$. degree in electrical engineering from the University of Melbourne, Melbourne, Australia, in 1992.

He has been with City University of Hong Kong since 2000, where he is now a Professor, and he was with School of Electrical Engineering, University of New South Wales, Australia, from 1992 to 1999. He was a Visiting Fellow with the National University of Singapore (1997) and Aachen Technology University, Germany (1997-1998). His current research interests include robust adaptive control, signal processing, piecewise linear systems, and intelligent systems and control.

Prof. Feng is an Associate Editor of the IEEE TRANSACTIONS ON AUTOMATIC CONTROL and the IEEE TRANSACTIONS ON FUZZY SYSTEMS and was an Associate Editor of the IEEE TRANSACTIONS ON SYSTEMS, MAN \& CYBERNETICS, PART C and Journal of Control Theory and Applications, and the Conference Editorial Board of IEEE Control System Society. He was awarded an Alexander von Humboldt Fellowship in 1997-1998 and the 2005 IEEE TRANSACTIONS ON FUZZY SYSTEMS Outstanding Paper Award in 2007.

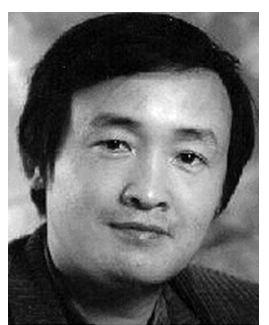

Xiaohui Liu received the B.Eng. degree in computing from Hohai University, Nanjing, China, in 1982 and the Ph.D. degree in computer science from Heriot-Watt University, Edinburg, U.K., in 1988.

$\mathrm{He}$ is a Professor of Computing with Brunel University, Middlesex, U.K. He leads the Intelligent Data Analysis (IDA) Group, performing interdisciplinary research involving artificial intelligence, dynamic systems, image and signal processing, and statistics, particularly for applications in biology, engineering and medicine. He serves on editorial boards of four computing journals, founded the biennial international conference series on IDA in 1995, and has given numerous invited talks in bioinformatics, data mining and statistics conferences. 